\title{
Totally Undecomposable Functions: Applications to Efficient Multiple-Valued Decompositions
}

\author{
Tsutomu Sasao \\ Department of Computer Science and Electronics \\ Kyushu Institute of Technology \\ Iizuka 820-8502, Japan
}

\begin{abstract}
A function $f: P^{n} \rightarrow P, P=\{0,1, \ldots, p-1\}$ is $k$ decomposable iff $f$ can be represented as $f\left(X_{1}, X_{2}\right)=$ $g\left(h_{1}\left(X_{1}\right), h_{2}\left(X_{1}\right), \ldots, h_{k}\left(X_{1}\right), X_{2}\right)$, where $\left(X_{1}, X_{2}\right)$ is a bipartition of input variables. This paper introduces the notion of totally $k$-undecomposable functions. By using this concept, we can drastically reduce the search space to find $k$-decompositions. A systematic method to find the bipartitions of input variables that will not produce any $k$-decompositions is presented. By combining it to the conventional decomposition methods, we can build an efficient functional decomposition system. This method is promising to design LUT-based FPGAs.

Key words: Functional decomposition, Symmetric function, LUT-based FPGA, Multiple-valued logic function.
\end{abstract}

\section{Introduction}

Decompositions of logic functions have been studied for many years. Major contributions are summarized as follows:

- Formulations using decomposition tables [1, 5].

- Formulations using compatibility [12].

- Fast method using Jacobian [20].

- Applications to multi-level PLA networks [13, 6].

- Extension to incompletely specified functions [21].

- Computation of column multiplicity using BDDs $[14,8,4]$.

- Application to FPGAs [11].

- Bi-decomposition [15].

- Fast method $[2,10]$.

- Extension to multiple-valued logic [7, 9].

- Extension to multiple-output functions [18, 22, 9].

In the above contributions, most are related to twovalued functions. However, extensions to multiplevalued functions are quite natural.

In this paper, we will consider decompositions shown in Fig. 1.1. Given a multiple-valued function $f: P^{n} \rightarrow P, P=\{0,1, \ldots, p-1\}$, we will consider the problem whether $f$ can be represented as $f\left(X_{1}, X_{2}\right)=$ $g\left(h_{1}\left(X_{1}\right), h_{2}\left(X_{1}\right), \ldots, h_{k}\left(X_{1}\right), X_{2}\right)$, or not.

Let $n$ be the number of the input variables, then we have to consider nearly $2^{n}$ different bipartitions $\left(X_{1}, X_{2}\right)$ of the input variables $\left\{x_{1}, x_{2}, \ldots, x_{n}\right\}$. When

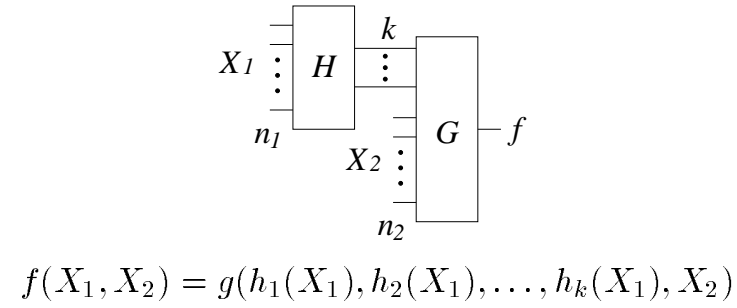

Figure 1.1: Disjoint $k$-decomposition.

$n$ is large, the number of bipartitions to consider is too large, and the exhaustive search is impractical.

This paper introduces the concept of totally undecomposable functions. By using this concept, we can drastically reduce computation time to find decompositions. This paper shows a systematic method to find the bipartitions of input variables that will not produce any decompositions. By combining it to the conventional decomposition methods, we can build an efficient functional decomposition system.

The rest of this paper is organized as follows: Section II gives definitions and basic properties of functional decompositions. Section III introduces the concept of $k$-undecomposable functions. It also derives a theorem to find bipartitions $\left(X_{1}, X_{2}\right)$ that will not produce any $k$-decomposition. Section IV shows a method to represent a set of bipartitions by using a switching function. Section $V$ enumerates the number of $k$-undecomposable functions. It also shows that, for sufficiently large $n$, almost all functions are totally $k$ undecomposable.

\section{Definitions and Basic Properties}

Definition 2.1 $A$ p-valued function is a mapping $f: P^{n} \rightarrow P$, where $P=\{0,1, \ldots, p-1\}$ and $p \geq 2$. If $p=2, f$ is a switching function.

Definition 2.2 Let the set of the input variables be $\{X\}=\left\{x_{1}, x_{2}, \ldots, x_{n}\right\} .\left(X_{1}, X_{2}, \ldots, X_{r}\right)$ is a partition of $X$ if $\left\{X_{i}\right\} \cap\left\{X_{j}\right\}=\phi(1 \leq i<j \leq n)$ and $\left\{X_{1}\right\} \cup\left\{X_{2}\right\} \cup \cdots \cup\left\{X_{r}\right\}=\{X\}$. Especially when $r=2$, the partition is a bipartition. The number of the variables in $\{X\}$ is denoted by $|X|$.

Definition 2.3 A p-valued function $f$ has a disjoint $k$-decomposition iff $f$ is represented as $f\left(X_{1}, X_{2}\right)=$ 


\begin{tabular}{ccccc|c|c|c|c|c|c|c|}
\multicolumn{10}{c|}{$X_{1}=\left(x_{1}, x_{2}\right)$} \\
$X_{2}=\left(x_{3}, x_{4}\right)$ & 0 & 0 & 0 & \multicolumn{1}{c|}{1} & 1 & 1 & 2 & 2 & 2 \\
00 & 0 & 1 & 2 & 0 & 1 & 2 & 0 & 1 & 2 \\
\cline { 2 - 11 } 01 & 0 & 1 & 1 & 2 & 2 & 2 & 1 & 1 & 1 \\
02 & 1 & 1 & 1 & 0 & 0 & 0 & 1 & 1 & 1 \\
\hline 10 & 0 & 0 & 0 & 0 & 0 & 0 & 0 & 0 & 0 \\
11 & 1 & 0 & 0 & 1 & 1 & 1 & 0 & 2 & 2 \\
12 & 2 & 0 & 0 & 2 & 2 & 2 & 0 & 2 & 2 \\
\hline 20 & 0 & 2 & 2 & 0 & 0 & 0 & 2 & 2 & 2 \\
21 & 1 & 2 & 2 & 1 & 1 & 1 & 2 & 0 & 0 \\
22 & 2 & 2 & 2 & 2 & 2 & 2 & 2 & 0 & 0 \\
\hline
\end{tabular}

Figure 2.1: Decomposition table.

$g\left(h_{1}\left(X_{1}\right), h_{2}\left(X_{1}\right), \ldots, h_{k}\left(X_{1}\right), X_{2}\right)$, where $\left(X_{1}, X_{2}\right)$ is a bipartition of $X$, and $g$ and $h_{i}$ are $p$-valued functions. If $\left|X_{1}\right| \geq k+1$ and $\left|X_{2}\right| \geq 1+\left\lceil\log _{p} k\right\rceil$, then the decomposition is non-trivial, and $f$ is $k$-decomposable, where $\lceil a\rceil$ denotes the least integer not smaller than a. We also assume that functions with up to two variables are decomposable. $\left\{X_{1}\right\}$ and $\left\{X_{2}\right\}$ are the bound set and the free set, respectively. Variables in $\left\{X_{1}\right\}$ and $\left\{X_{2}\right\}$ are bound variables and free variables, respectively. When $f$ is $k$-decomposable, $f$ is realized by the network shown in Fig. 1.1.

Definition 2.4 If $f$ does not depend on one or more variables, then $f$ is degenerate.

Note that if $f$ is degenerate, then $f$ is decomposable.

Definition 2.5 Let $f(X)$ be a p-valued function, and $\left(X_{1}, X_{2}\right)$ be a bipartition of $X$, where $n_{1}=\left|X_{1}\right|$ and $n_{2}=\left|X_{2}\right|$. The decomposition table of $f$ has $p^{n_{1}}$ columns and $p^{n_{2}}$ rows, each column has distinct $p$-ary label of $n_{1}$ digits, each row has distinct $p$-ary label of $n_{2}$ digits, and the corresponding entry of the table represents the value of $f$.

Example 2.1 Let $f(X)$ be a function $f:\{0,1,2\}^{4} \rightarrow$ $\{0,1,2\}$, and $\left(X_{1}, X_{2}\right)$ be a bipartition of $X$, where $X_{1}=\left(x_{1}, x_{2}\right)$ and $X_{2}=\left(x_{3}, x_{4}\right)$. Fig. 2.1 is an example of a decomposition table.

Definition 2.6 The number of different column patterns in the decomposition table for a bipartition $\left(X_{1}, X_{2}\right)$ is the column multiplicity and is denoted by $\mu\left(f: X_{1}, X_{2}\right)$.

Theorem 2.1 A p-valued function $f(X)$ has a disjoint $k$-decomposition $f(X)=g\left(h_{1}\left(X_{1}\right), h_{2}\left(X_{1}\right), \ldots\right.$, $\left.h_{k}\left(X_{1}\right), X_{2}\right)$ iff $\mu\left(f: X_{1}, X_{2}\right) \leq p^{k}$.

The size of decomposition tables for $n$ variables is $p^{n}$, and the number of different bipartitions is $O\left(2^{n}\right)$. Thus, the straightforward method to find a $k$-decomposition is impractical for the functions with many inputs. A method to find decompositions by using ROBDDs (reduced ordered binary decision diagrams) or ROMDDs (reduced ordered multi-valued decision diagrams) has been developed.

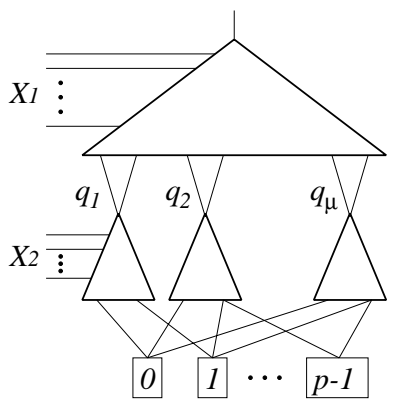

Figure 2.2: Computation of column multiplicity $\mu(f$ : $\left.X_{1}, X_{2}\right)$.

Theorem 2.2 [14, 8, 7] Let $\left(X_{1}, X_{2}\right)$ be a bipartition of $X$. Suppose that the ROMDD for $f(X)$ is partitioned into two blocks as shown in Fig. 2.2. The number of nodes in the lower block that are adjacent to the upper block is equal to $\mu\left(f: X_{1}, X_{2}\right)$.

Lemma 2.1 For any bipartition $\left(X_{1}, X_{2}\right)$ of input variables of a $p$-valued function $f, 1 \leq \mu\left(f: X_{1}, X_{2}\right) \leq$ $\min \left(p^{n_{1}}, p^{p^{n_{2}}}\right)$, where $n_{1}=\left|X_{1}\right|$ and $n_{2}=\left|X_{2}\right|$.

(Proof) The number of columns in the decomposition table is $p^{n_{1}}$. Thus, we have $\mu\left(f: X_{1}, X_{2}\right) \leq p^{n_{1}}$. The number of different functions of $n_{2}$ variables is $p^{p^{n_{2}}}$. Since each column of the decomposition table shows an $n_{2}$-variable function, we have $\mu\left(f: X_{1}, X_{2}\right) \leq p^{p^{n_{2}}}$.

Definition 2.7 Let $f\left(X_{A}, X_{B}\right)$ be a function, where $\left|X_{B}\right|=n_{B}$. Let $\vec{a}_{B} \in P^{n_{B}}$ be an assignment for $X_{B}$. Then, $f\left(X_{A}, \vec{a}_{B}\right)$ denotes the sub-function, where the values of $X_{B}$ are fixed to the constants $\vec{a}_{B} \cdot f\left(\vec{a}_{A}, X_{B}\right)$ is similarly defined.

Definition 2.8 Let $X=\left(x_{1}, x_{2}, \ldots, x_{n}\right)$ and $\vec{a}=$ $\left(a_{1}, a_{2}, \ldots, a_{n}\right)$. Then,

$$
X^{\vec{a}}\left\{\begin{array}{l}
=p-1 \text { if } x_{i}=a_{i} \text { for } i=1,2, \ldots, n . \\
=0 \text { otherwise. }
\end{array}\right.
$$

Lemma 2.2 If $k \geq p^{\left|X_{2}\right|}$, then any $p$-valued function is realized in the network shown in Fig. 1.1.

(Proof) Let $n_{2}=\left|X_{2}\right|$. An arbitrary function $f\left(X_{1}, X_{2}\right)$ is represented by $f\left(X_{1}, X_{2}\right)=$ $\bigvee_{\vec{a} \in P^{n_{2}}} f\left(X_{1}, \vec{a}\right) X_{2}^{\vec{a}}$, where $P=\{0,1, \ldots, p-1\}$. Since the number of products in the above expression is at most $p^{n_{2}}$, we have the lemma.

Example 2.2 When $n_{2}=1$ and $k=p$, any $p$-valued function is realized in the network shown in Fig. 2.3 by using the following expansion:

$f\left(X_{1}, X_{2}\right)=X_{2}^{0} f_{0}\left(X_{1}\right) \vee X_{2}^{1} f_{1}\left(X_{1}\right) \vee \cdots \vee X_{2}^{p-1} f_{p-1}\left(X_{1}\right)$. 


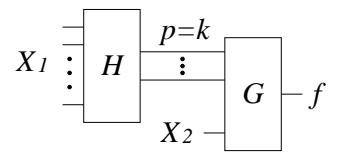

Figure 2.3: Example of a trivial $k$-decomposition.

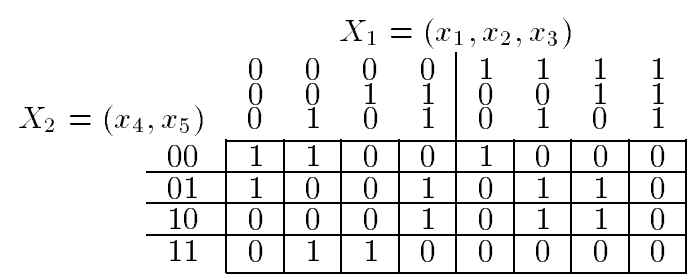

Figure 3.1: Totally 2-undecomposable function.

We assume that the $k$-decomposition in Lemma 2.2 is trivial. This is why we assumed that $n_{2} \geq\left\lceil\log _{p} k\right\rceil+1$ in Definition 2.3.

\section{III $k$-Undecomposable Functions}

In this part, we introduce the notion of totally $k$-undecomposable functions. We will show that if $f\left(X_{A}, \vec{a}_{B}\right)$ is totally $k$-undecomposable, then $f\left(X_{A}, X_{B}\right)$ is $k$-undecomposable for many bipartitions. Thus, by finding totally $k$-undecomposable subfunctions, we can drastically reduce the search space for functional decompositions.

Definition $3.1 A$ p-valued function $f(X)$ is totally $k$-undecomposable if $\mu\left(f: X_{1}, X_{2}\right)>p^{k}$ for any bipartition $\left(X_{1}, X_{2}\right)$, where $\left|X_{1}\right| \geq 1+k$ and $\left|X_{2}\right| \geq$ $1+\left\lceil\log _{p} k\right\rceil$.

Example 3.1 Consider the case where $n=3, p=2$, and $k=1$. $f\left(x_{1}, x_{2}, x_{3}\right)=x_{1} x_{2} \vee x_{2} x_{3} \vee x_{3} x_{1}$ is totally 1-undecomposable.

Example 3.2 Consider the case where $n=5, p=2$, and $k=2$. A five-variable function $f$ shown in Fig. 3.1 is totally 2-undecomposable, since $\mu\left(f: X_{1}, X_{2}\right)>4$ for any bipartitions with $\left|X_{1}\right|=3$ and $\left|X_{2}\right|=2$.

Lemma 3.1 Let $\left(X_{1 A}, X_{1 B}, X_{2 A}, X_{2 B}\right)$ be a partition of $X$, where $\left|X_{1 A}\right| \geq k+1$ and $\left|X_{2 A}\right| \geq 1+$ $\left\lceil\log _{p} k\right\rceil$. Let $\vec{a}_{1 B}$ and $\overrightarrow{\vec{a}}_{2 B}$ be assignments of $X_{1 B}$ and $X_{2 B}$, respectively. If $f\left(X_{1 A}, \vec{a}_{1 B}, X_{2 A}, \vec{a}_{2 B}\right)$ has no $k$ decomposition of the form

$$
\begin{aligned}
\hat{f}\left(X_{1 A}, X_{2 A}\right) & =f\left(X_{1 A}, \vec{a}_{1 B}, X_{2 A}, \vec{a}_{2 B}\right) \\
& =\hat{g}\left(\hat{h}_{1}\left(X_{1 A}\right), \hat{h}_{2}\left(X_{1 A}\right), \ldots, \hat{h}_{k}\left(X_{1 A}\right), X_{2 A}\right),
\end{aligned}
$$

then, $f\left(X_{1 A}, X_{1 B}, X_{2 A}, X_{2 B}\right)$ has no $k$-decomposition of the form

$$
f\left(X_{1 A}, X_{1 B}, X_{2 A}, X_{2 B}\right)
$$

$$
\begin{aligned}
= & g\left(h_{1}\left(X_{1 A}, X_{1 B}\right), h_{2}\left(X_{1 A}, X_{1 B}\right), \ldots,\right. \\
& \left.h_{k}\left(X_{1 A}, X_{1 B}\right), X_{2 A}, X_{2 B}\right) .
\end{aligned}
$$

In this case, $\left\{X_{1 B}\right\}$ or $\left\{X_{2 B}\right\}$ can be empty set( $(s)$.

(Proof) Assume that $f$ has a $k$-decomposition of the form

$$
\begin{aligned}
f & \left(X_{1 A}, X_{1 B}, X_{2 A}, X_{2 B}\right) \\
= & g\left(h_{1}\left(X_{1 A}, X_{1 B}\right), h_{2}\left(X_{1 A}, X_{1 B}\right), \ldots,\right. \\
& \left.h_{k}\left(X_{1 A}, X_{1 B}\right), X_{2 A}, X_{2 B}\right) .
\end{aligned}
$$

Assign $\vec{a}_{1 B}$ and $\vec{a}_{2 B}$ to $X_{1 B}$ and $X_{2 B}$, respectively. Then, we have the decomposition $f\left(X_{1 A}, \vec{a}_{1 B}, X_{2 A}\right.$, $\left.\vec{a}_{2 B}\right)=g\left(h_{1}\left(X_{1 A}, \vec{a}_{1 B}\right), h_{2}\left(X_{1 A}, \vec{a}_{1 B}\right), \ldots, h_{k}\left(X_{1 A}\right.\right.$, $\left.\left.\vec{a}_{1 B}\right), X_{2 A}, \vec{a}_{2 B}\right)$. However, this contradicts the assumption of the lemma.

Example 3.3 Consider the case where $n=8, k=$ 2 , and $p=2$. Let $f\left(x_{1}, x_{2}, \ldots, x_{8}\right)$ be an 8variable function. If $\left(x_{1}, x_{2}, x_{3}, 0,1, x_{6}, x_{7}, 1\right)$ has no 2-decomposition of the form $\hat{f}\left(x_{1}, x_{2}, x_{3}, x_{6}, x_{7}\right)=$ $\hat{g}\left(\hat{h}_{1}\left(x_{1}, x_{2}, x_{3}\right), \hat{h}_{2}\left(x_{1}, x_{2}, x_{3}\right), x_{6}, x_{7}\right)$, then $f\left(x_{1}, x_{2}\right.$, $\left.\ldots, x_{8}\right)$ has no 2-decomposition of the form $f=$ $g\left(h_{1}\left(x_{1}, x_{2}, x_{3}, x_{4}, x_{5}\right), h_{2}\left(x_{1}, x_{2}, x_{3}, x_{4}, x_{5}\right), x_{6}, x_{7}, x_{8}\right)$. In this example, $X_{1 A}=\left(x_{1}, x_{2}, x_{3}\right), X_{1 B}=\left(x_{4}, x_{5}\right)$, $X_{2 A}=\left(x_{6}, x_{7}\right), X_{2 B}=\left(x_{8}\right), \vec{a}_{1 B}=(0,1)$, and $\vec{a}_{2 B}=0$.

Theorem 3.1 Let $\left(X_{A}, X_{B}\right)$ be a partition of $X$, where $\left|X_{A}\right| \geq k+\left\lceil\log _{p} k\right\rceil+2$ and $\left|X_{B}\right| \geq 1$. For an assignment $\vec{a}_{B}$, if $f\left(X_{A}, \vec{a}_{B}\right)$ is totally $k$-undecomposable, then $f$ has no decomposition of the form $f\left(X_{1}, X_{2}\right)=$ $g\left(h_{1}\left(X_{1}\right), h_{2}\left(X_{1}\right), \ldots, h_{k}\left(X_{1}\right), X_{2}\right)$, where $\left(X_{1}, X_{2}\right)$ is a bipartition of $X,\left|\left\{X_{A}\right\} \cap\left\{X_{1}\right\}\right| \geq k+1$, and $\left|\left\{X_{A}\right\} \cap\left\{X_{2}\right\}\right| \geq 1+\left\lceil\log _{p} k\right\rceil$.

(Proof) Let $X_{A}=\left(X_{1 A}, X_{2 A}\right)$ and $X_{B}=\left(X_{1 B}, X_{2 B}\right)$. Then, apply Lemma 3.1, and we have the theorem. $\square$

Definition 3.2 Let $\left(X_{1}, X_{2}\right)$ be a bipartition of $\left\{x_{1}, x_{2}, \ldots, x_{n}\right\}$, where $X_{1}=\left(x_{1}, x_{2}, \ldots, x_{r}\right)$ and $X_{2}=$ $\left(x_{r+1}, x_{r+2}, \ldots, x_{n}\right)$. Such a bipartition is compactly denoted by the bipartition of integers $(1,2, \ldots, r \mid r+$ $1, r+2, \ldots, n)$.

Example 3.4 Let $f\left(x_{1}, x_{2}, x_{3}, x_{4}, x_{5}\right)$ be a five-variable two-valued function. If $f\left(x_{1}, x_{2}, x_{3}, 0,0\right)$ is totally 1-undecomposable, then $f$ is 1-undecomposable for the following 12 bipartitions:

$(1,2 \mid 3,4,5),(1,2,4 \mid 3,5),(1,2,5 \mid 3,4),(1,2,4,5 \mid 3)$, $(1,3 \mid 2,4,5),(1,3,4 \mid 2,5),(1,3,5 \mid 2,4),(1,3,4,5 \mid 2)$, $(2,3 \mid 1,4,5),(2,3,4 \mid 1,5),(2,3,5 \mid 1,4),(2,3,4,5 \mid 1)$. 
Theorem 3.2 Consider a p-valued function $f\left(X_{A}, X_{B}\right)$, where $n_{A}=\left|X_{A}\right| \geq k+\left\lceil\log _{p} k\right\rceil+2$ and $n_{B}=\left|X_{B}\right| \geq 1$. For an assignment $\vec{a}_{B}$, if $f\left(X_{A}, \vec{a}_{B}\right)$ is totally $k$-undecomposable, then $f$ is $k$-undecomposable for

$$
\alpha\left(n_{A}, n_{B}, p, k\right)=\left[\sum_{i=k+1}^{n_{A}-1-\left\lceil\log _{p} k\right\rceil} C\left(n_{A}, i\right)\right] 2^{n_{B}}
$$

bipartitions.

(Proof) $F$ is $k$-undecomposable when the following conditions are satisfied:

1) More than $k$ variables in $\left\{X_{A}\right\}$ are included as bound variables.

2) More than $\left\lceil\log _{p} k\right\rceil$ variables in $\left\{X_{A}\right\}$ are included as free variables.

3) Variables in $\left\{X_{B}\right\}$ can be either in the bound set or the free set.

From 1) and 2), we have the first factor. And, from 3), we have the second factor.

Example 3.5 Let $f\left(x_{1}, x_{2}, x_{3}, x_{4}, x_{5}\right)$ be a five-variable 2-valued function. If $f\left(x_{1}, x_{2}, x_{3}, 0,0\right)$ is totally 1-undecomposable, then $f$ is 1-undecomposable for $\alpha=$ 12 bipartitions, since $k=1, p=2, n_{1}=3$, and $n_{2}=2$. This is also verified by Example 3.4

Corollary 3.1 Consider an n-variable function $f\left(X_{A}, X_{B}\right)$, where $n_{A}=\left|X_{A}\right| \geq k+\left\lceil\log _{p} k\right\rceil+2$, and $n_{B}=\left|X_{B}\right| \geq 1$. For an assignment $\vec{a}_{B}$, if $\left(X_{A}, \vec{a}_{B}\right)$ is totally $k$-undecomposable, then we have to check for at most

$\beta\left(n_{A}, n_{B}, p, k\right)=\left[\sum_{i=0}^{k} C\left(n_{A}, i\right)+\sum_{j=0}^{\left\lceil\log _{p} k\right\rceil} C\left(n_{A}, j\right)\right] 2^{n_{B}}$

bipartitions.

(Proof) There are $2^{n}=2^{n_{A}} 2^{n_{B}}$ bipartitions. Among them, $\alpha\left(n_{A}, n_{B}, p, k\right)$ bipartitions are $k$ undecomposable. So, we have to check at most $\beta\left(n_{A}, n_{B}, p, k\right)=2^{n}-\alpha\left(n_{A}, n_{B}, p, k\right)$ bipartitions.

Example 3.6 Corollary 3.1 shows that when $p=2$ and $k=1$, the fraction of $\beta$ to $2^{n}$ is $\gamma=\frac{\beta}{2^{n}}=\frac{n_{A}+2}{2^{n} A}$. Therefore, when $n_{A}=3, \gamma=5 / 8$; when $n_{A}=4$, $\gamma=3 / 8$; when $n_{A}=5, \gamma=7 / 32$; and when $n_{A}=6$, $\gamma=1 / 8$

\section{Switching Function Representing Set of Bipartitions}

Functional decomposition is to find a bipartition $\left(X_{1}, X_{2}\right)$ such that $f\left(X_{1}, X_{2}\right)=g\left(h_{1}\left(X_{1}\right), h_{2}\left(X_{1}\right), \ldots\right.$, $\left.h_{k}\left(X_{1}\right), X_{2}\right)$. There are $2^{n}$ different bipartitions including trivial ones, and these can be represented by a switching function of $n$ variables. In this part, we will introduce such representations. Also, bipartitions that will not produce decompositions are compactly denoted by symmetric functions. We also introduce notations for symmetric functions.

Definition 4.1 A function $f$ is a totally symmetric function if any permutation of the variables in $f$ does not change the function.

Definition 4.2 The elementary symmetric functions of $n$ variables are

$S_{0}^{n}=\bar{x}_{1} \bar{x}_{2} \cdots \bar{x}_{n}$,

$S_{1}^{n}=x_{1} \bar{x}_{2} \cdots \bar{x}_{n} \vee \bar{x}_{1} x_{2} \bar{x}_{3} \cdots \bar{x}_{n} \vee \cdots \vee \bar{x}_{1} \bar{x}_{2} \cdots \bar{x}_{n-1} x_{n}$, ............... and

$S_{n}^{n}=x_{1} x_{2} \cdots x_{n}$.

$S_{i}^{n}=1$ iff exactly $i$ inputs are equal to one. Let $A \subseteq$ $\{0,1, \ldots, n\} . A$ symmetric function $S_{A}^{n}$ is defined as follows:

$$
S_{A}^{n}=\bigvee_{i \in A} S_{i}^{n}
$$

Example 4.1 $f\left(x_{1}, x_{2}, x_{3}\right)=x_{1} x_{2} x_{3} \vee x_{1} \bar{x}_{2} \bar{x}_{3} \vee$ $\bar{x}_{1} x_{2} \bar{x}_{3} \vee \bar{x}_{1} \bar{x}_{2} x_{3}$ is a totally symmetric function. $f=1$ when all the variables are one, or when only one variable is one. Thus, $f$ can be written as $S_{1}^{3} \vee S_{3}^{3}=S_{\{1,3\}}^{3}$.

Definition 4.3 A set of bipartitions of the input variables $\left\{x_{1}, x_{2}, \ldots, x_{n}\right\}$ is represented by a switching function $b p$ of $n$ variables. In $b p, x_{i}=1$ denotes that $x_{i}$ is in the bound set, and $x_{i}=0$ denotes that $x_{i}$ is in the free set. The number of true minterms of bp is denoted by $|b p|$.

Example 4.2 Suppose that $n=5$. The minterm $x_{1} x_{2} x_{3} \bar{x}_{4} \bar{x}_{5}$ denotes that $x_{1}, x_{2}$, and $x_{3}$ are in the bound set, and $x_{4}$ and $x_{5}$ are in the free set.

Lemma 4.1 The set of bipartitions for trivial $k$ decompositions for n-variable p-valued function is given by

$$
u_{0}=S_{\{0,1, \ldots, k\}}^{n} \vee S_{\left\{n-\left\lceil\log _{p} k\right\rceil, \ldots, n\right\}}^{n} .
$$

(Proof) When the number of variables in the bound set is less than $k+1$, then it is a trivial decomposition. To be non-trivial $k$-decomposition, at least $1+\left\lceil\log _{p} k\right\rceil$ variables must be in the free set. So, if the number of variables in the bound set is greater than $n-1-$ $\left\lceil\log _{p} k\right\rceil$, then it is a trivial decomposition.

Example 4.3 Let $n=10, p=2$, and $k=2$, then the set of bipartitions for trivial $k$-decompositions is given by $u_{0}=S_{\{0,1,2,9,10\}}^{10}$. This is explained as follows: If the number of bound variables is two or smaller, then 
the decomposition is trivial, since the module for $H$ has two outputs. If the number of variables in the bound set is 9 or 10 , then the number of free variables is one or zero. By Definition 2.3, this also corresponds to a trivial decomposition. Thus, the number of trivial decompositions is given by

$$
\begin{aligned}
\left|u_{0}\right|= & C(10,0)+C(10,1)+C(10,2) \\
& +C(10,9)+C(10,10)=67 .
\end{aligned}
$$

The set of non-trivial bipartitions is given by $\bar{u}_{0}=$ $S_{\{3,4,5,6,7,8\}}^{10}$.

Theorem 4.1 Let $f(X)$ be a p-valued function, and $\left(X_{A}, X_{B}\right)$ be a partition of $X$. If $f\left(X_{A}, \vec{a}_{B}\right)=$ $\hat{f}\left(x_{1}, x_{2}, \ldots, x_{r}\right)$ is totally $k$-undecomposable, then $f$ has no $k$-decomposition for the bipartitions

$$
u=S_{\left\{k+1, k+2, \ldots, r-1-\left\lceil\log _{p} k\right\rceil\right\}}^{r}\left(x_{1}, x_{2}, \ldots, x_{r}\right) .
$$

(Proof) By Theorem 3.1 and Lemma 4.1, $f$ is $k$ undecomposable for these bipartitions.

Example 4.4 Consider the case where $p=2, n=5$, $k=1$ and $r=3$. If $f\left(x_{1}, x_{2}, x_{3}, 0,0\right)$ is totally 1 undecomposable, then $f$ is undecomposable for the bipartitions

$$
u=S_{2}^{3}\left(x_{1}, x_{2}, x_{3}\right)=\bar{x}_{1} x_{2} x_{3} \vee x_{1} \bar{x}_{2} x_{3} \vee x_{1} x_{2} \bar{x}_{3} .
$$

Note that $u$ denotes the same set of bipartitions as Example 3.4.

Example 4.5 Suppose that we have to check whether the given 2-valued 10-variable function $f\left(x_{1}, x_{2}, \ldots\right.$, $x_{10}$ ) can be realized by a network shown in Fig. 4.1. In this case, the straightforward method needs to check all possible bipartitions $\left(X_{1}, X_{2}\right)$, where $\left|X_{1}\right|=5$ and $\left|X_{2}\right|=5$. This set of bipartitions is represented by $S_{5}^{10}\left(x_{1}, x_{2}, \ldots, x_{10}\right)$, and the number of bipartitions to consider is $\left|S_{5}^{10}\left(x_{1}, x_{2}, \ldots, x_{10}\right)\right|=C(10,5)=252$. However, if $f\left(x_{1}, x_{2}, x_{3}, x_{4}, x_{5}, 0,0,0,0,0\right)$ is totally 2 undecomposable, then we need not check for $C(5,3) \times$ $C(5,2)=100$ bipartitions. This fact is explained as follows: From Theorem 4.1, the set of bipartitions that will not produce 2-decomposition is given by

$$
u_{1}=S_{3}^{5}\left(x_{1}, x_{2}, x_{3}, x_{4}, x_{5}\right) S_{2}^{5}\left(x_{6}, x_{7}, x_{8}, x_{9}, x_{10}\right) .
$$

In $u_{1}$, the first factor selects three variables from $\left\{x_{1}, x_{2}, x_{3}, x_{4}, x_{5}\right\}$ as bound variables, and the second factor selects two variables from $\left\{x_{6}, x_{7}, x_{8}, x_{9}, x_{10}\right\}$ as bound variables. For example, suppose that $\left\{X_{1}\right\}=\left\{x_{1}, x_{2}, x_{3}, x_{6}, x_{7}\right\}$ is selected as a bound set, and $\left\{X_{2}\right\}=\left\{x_{4}, x_{5}, x_{8}, x_{9}, x_{10}\right\}$ is selected as a free set. This bipartition $\left(X_{1}, X_{2}\right)$ does not produce 2-decomposition, since $\left\{x_{1}, x_{2}, x_{3}\right\}$ is in the bound set and $\left\{x_{4}, x_{5}\right\}$ is in the free

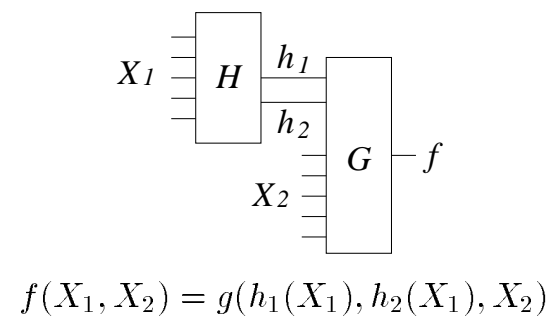

Figure 4.1: 2-decomposition of 10-variable function.

set, and $f\left(x_{1}, x_{2}, x_{3}, x_{4}, x_{5}, 0,0,0,0,0\right)$ is totally 2undecomposable. Note that $\left|u_{1}\right|=C(5,3) C(5,2)=$ $10 \times 10=100$.

In a similar way, if $f\left(0,0,0,0,0, x_{6}, x_{7}, x_{8}, x_{9}, x_{10}\right)$ is also totally 2-undecomposable, then the following bipartitions need not be checked:

$$
u_{2}=S_{3}^{5}\left(x_{6}, x_{7}, x_{8}, x_{9}, x_{10}\right) S_{2}^{5}\left(x_{1}, x_{2}, x_{3}, x_{4}, x_{5}\right) .
$$

$u_{2}$ denotes $C(5,3) \times C(5,2)=100$ bipartitions for that no 2-decomposition exist. So, we need only to check for the following bipartitions:

$$
b p=S_{5}^{10}\left(x_{1}, x_{2}, \ldots, x_{10}\right) \overline{u_{1}} \overline{u_{2}} .
$$

Since $u_{1}$ and $u_{2}$ are mutually disjoint, we have only to check

$$
\begin{aligned}
|b p| & =\left|S_{5}^{10}\left(x_{1}, x_{2}, \ldots, x_{10}\right)\right|-\left|u_{1}\right|-\left|u_{2}\right| \\
& =C(10,5)-100-100=52
\end{aligned}
$$

bipartitions. In this case, we can reduce the search space into one fifth by finding two subfunctions that are 2-undecomposable.

Theorem 4.2 Let $f\left(x_{1}, x_{2}, \ldots, x_{n-1}, a\right)$ be totally $k$ undecomposable, where $a \in P$. Then, $f\left(x_{1}, x_{2}, \ldots\right.$, $\left.x_{n-1}, x_{n}\right)$ is totally $k$-undecomposable iff $f$ is undecomposable for the following $C\left(n-1,\left\lceil\log _{p} k\right\rceil\right)+C(n-1, k)$ bipartitions: $\quad S_{\left\{n-1-\left\lceil\log _{p} k\right\rceil\right\}}^{n-1}\left(x_{1}, x_{2}, \ldots, x_{n-1}\right) \bar{x}_{n} \vee$ $S_{k}^{n-1}\left(x_{1}, x_{2}, \ldots, x_{n-1}\right) x_{n}$.

Example 4.6 Consider the case where $n=6, p=2$, and $k=2$. Suppose that $f\left(x_{1}, x_{2}, x_{3}, x_{4}, x_{5}, 0\right)$ is totally $k$-undecomposable. To show that $f$ is totally undecomposable, we need the followings: For $x_{6}=$ 0 , we have to check for the bipartitions denoted by $S_{4}^{5}\left(x_{1}, x_{2}, x_{3}, x_{4}, x_{5}\right) \bar{x}_{6}$. Fig. 4.2(a) shows an example of $C(5,4)$ bipartitions. For $x_{6}=1$, we have to check for bipartitions denoted by $S_{2}^{5}\left(x_{1}, x_{2}, x_{3}, x_{4}, x_{5}\right) x_{6}$. Fig. 4.2(b) shows an example of $C(5,2)=10$ bipartitions. 


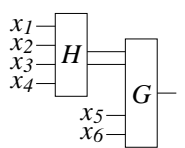

(a) 2-decomposition corresponding to $\bar{x}_{6}$.

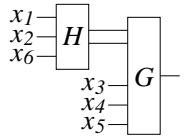

(b) 2-decomposition corresponding to $x_{6}$.
Figure 4.2:

\section{Number of Totally}

\section{$k$-Undecomposable Functions}

When $n$ is sufficiently large, almost all functions are totally 1-undecomposable [19]. In this part, we will show that almost all functions are also totally $k$ undecomposable.

\section{1 p-valued case}

Lemma 5.1 Let $N_{d}(n, p, k)$ be the number of $n$ variable p-valued $k$-decomposable functions. Then,

$$
N_{d}(n, p, k) \leq \sum_{n_{1}=k+1}^{n-1-\left\lceil\log _{p} k\right\rceil} C\left(n, n_{1}\right) p^{k p^{n_{1}}+p^{n-n_{1}+k}} .
$$

(Proof) Suppose that a function $f$ has a disjoint $k$ decomposition shown in Fig. 1.1. First, consider the module $H$. The number of ways to select bound variables is $C\left(n, n_{1}\right)$. Since $H$ has $n_{1}$ inputs and $k$ outputs, the number of functions for $H$ is $p^{k p^{n_{1}}}$. Next, consider the module $G$. Since $G$ has $n_{2}+k$ inputs and single output, the number of functions for $G$ is $p^{p^{n_{2}+k}}=p^{p^{n-n_{1}+k}}$. Hence, we have the lemma.

Theorem 5.1 Let $N_{u d}(n, p, k)$ be the number of $n$ variable $p$-valued totally $k$-undecomposable functions. Then,

$$
\frac{N_{u d}(n, p, k)}{p^{p^{n}}} \rightarrow 1 \text { as } n \rightarrow \infty .
$$

(Proof) Since $N_{u d}(n, p, k)+N_{d}(n, p, k)=p^{p^{n}}$, we will show that

$$
\frac{N_{d}(n, p, k)}{p^{p^{n}}} \rightarrow 0 \text { as } n \rightarrow \infty .
$$

From Lemma 5.1, we have

$$
N_{d}(n, p, k) \leq \sum_{n_{1}=k+1}^{n-1-\left\lceil\log _{p} k\right\rceil} C\left(n, n_{1}\right) p^{k p^{n_{1}}+p^{n_{2}+k}}
$$

Since $C\left(n, n_{1}\right)<2^{n}$, the right-hand-side of (5.2) is less than

$$
2^{n} \sum_{n_{1}=k+1}^{n-1-\left\lceil\log _{p} k\right\rceil} p^{k p^{n_{1}}+p^{n_{2}+k}}=2^{n} \sum_{n_{1}=k+1}^{n-1-\left\lceil\log _{p} k\right\rceil} p^{A\left(n_{1}\right)},
$$

where $A\left(n_{1}\right)=k p^{n_{1}}+p^{n_{2}+k}$. Note that $A\left(n_{1}\right)$ takes its maximum when $n_{1}=k+1$ and $n_{1}=n-1-\left\lceil\log _{p} k\right\rceil$, and the values of $A\left(n_{1}\right)$ are $k p^{k+1}+p^{n-1}$ and $p^{n-1}+$ $k p^{k+1}$, respectively. Thus, $A\left(n_{1}\right) \leq p^{n-1}+C$, where $C$ does not depend on $n$. So, (5.3) is less than

$$
D(n)=2^{n}\left(n-1-\left\lceil\log _{p} k\right\rceil-(k+1)\right) p^{p^{n-1}+C} .
$$

Let us take the logarithm of $D(n)$, and we have

$\log _{p} D(n)=n \log _{p} 2+\log _{p}\left(n-k-\left\lceil\log _{p} k\right\rceil-2\right)+p^{n-1}+C$.

Since $\frac{\log _{p} D(n)}{p^{n}} \rightarrow \frac{1}{p}$ as $n \rightarrow \infty$, we can conclude that (5.1) holds.

\subsection{Two-valued case}

When $n \geq 4$ and $p=2$, most functions are totally 1-undecomposable. For $k=1$, we obtained the values of $N_{u d}(n, p, k)$ by exhaustive enumeration:

$$
\begin{aligned}
& N_{u d}(3,2,1)=104, \\
& N_{u d}(4,2,1)=57,240, \text { and } \\
& N_{u d}(5,2,1)=4,290,002,448 .
\end{aligned}
$$

When $n=5$, there are $2^{2^{5}}=2^{32}=4,294,967,296$ functions. Thus, $99.9 \%$ of the functions are totally 1-undecomposable. The case of $k=2$ is interesting, since some FPGAs have LUTs with two outputs [3]. The decompositions must satisfy the relation:

$$
\left|X_{1}\right| \geq k+1 \text { and }\left|X_{2}\right| \geq 1+\left\lceil\log _{2} k\right\rceil \text {. }
$$

This requires that $|X|=\left|X_{1}\right|+\left|X_{2}\right| \geq k+2+\left\lceil\log _{2} k\right\rceil$. Thus, when $k=2$, only the functions with $n \geq 5$ are interesting. For $n=5$, the only case is $n_{1}=3$ and $n_{2}=2$, and we have $N_{u d}(5,2,2)=3,744,402,432$. Thus, $87.2 \%$ of the 5 -variable functions are totally 2 undecomposable.

\section{Conclusion and Comments}

In this paper, we defined totally $k$-undecomposable logic functions, and showed a systematic method to find a set of bipartitions that will not produce disjoint $k$-decompositions. Key contributions are:

1) Generation of a set of $k$-undecomposable bipartitions from totally $k$-undecomposable subfunctions.

2) Representation of $k$-undecomposable bipartitions by an $n$-variable switching function.

3) Enumeration of totally $k$-undecomposable functions.

The presented method can be extended to the case of incompletely specified functions. This method can be combined to existing decomposition methods to reduce search space. 
When $n=3$ or $4, p=2$ and $k=1$, totally $k$ undecomposable functions are easily detected by BDDs and look-up tables [17]. By using this method, we can show the undecomposability of randomly generated functions very quickly.

We decomposed more than four thousand benchmark functions including functions with 256 inputs and 245 outputs $[16,17]$. Experimental results for $p=2$ and $k=1$ show that for 1-undecomposable functions, the computation time were reduced to up to one hundreds. Currently, we are developing a system for $k$ decompositions with $k=2$.

Even if the given functions have two-valued inputs only, functional decompositions with multi-valued inputs seems to be useful. This is explained as follows: Suppose that a completely specified two-valued input function has a $k$-decomposition of the form $f\left(X_{1}, X_{2}\right)=g\left(h_{1}\left(X_{1}\right), g_{2}\left(X_{1}\right), \ldots, g_{k}\left(X_{1}\right), X_{2}\right)$, and that $\mu\left(f: X_{1}, X_{2}\right)<2^{k}$. In this case, assigning $\mu\left(f: X_{1}, X_{2}\right)$ different binary vectors to the $k$ outputs of $H$ produces don't care conditions for function $g$. This makes decomposition problem very difficult [21]. However, if we do not assign the binary vectors to the output of $h$, but assume that $h$ produces a multiplevalued output, then no don't cares are generated. In this case, the decomposition problem is easier.

\section{Acknowledgments}

This work was supported in part by a Grant in Aid for Scientific Research of the Ministry of Education, Science, Culture and Sports of Japan. Constructive comments of the reviewers improved the readability.

\section{References}

[1] R. L. Ashenhurst, "The decomposition of switching functions," In Proceedings of an International Symposium on the Theory of Switching, pp. 74-116, April 1957.

[2] V. Bertacco and M. Damiani, "The disjunctive decomposition of logic functions," Proc. ICCAD, pp. 78-82, 1997.

[3] S. D. Brown, R. J. Francis, J. Rose, and Z. G. Vranesic, Field Programmable Gate Arrays, Kluwer Academic Publishers, Boston 1992.

[4] S-C. Chang, M. Marek-Sadowska, and T. Hwang, "Technology mapping for LUT FPGA's based on decomposition of binary decision diagrams," IEEE Trans. CAD, Vol. CAD-15, No. pp. 1226-1236, Oct. 1996.

[5] H. A. Curtis, A New Approach to the Design of Switching Circuits, D. Van Nostrand Co., Princeton, NJ, 1962.

[6] S. Devadas, A. Wang, A. R. Newton, and A. Sangiovanni-Vincentelli, "Boolean decomposition in multilevel logic optimization," IEEE Journal of Solid-State Circuits, Vol. 24, pp. 399-408, April 1989.
[7] C. Files, R. Drechsler, and M. Perkowski, "Functional decomposition of MVL functions using multi-valued decision diagrams," ISMVL, pp. 27-32, May 1997.

[8] Y-T. Lai, M. Pedram, and S. B. K. Vrudhula, "EVBDD-based algorithm for integer linear programming, spectral transformation, and functional decomposition," IEEE Trans. CAD, Vol. 13, No. 8, pp. 959975, Aug. 1994.

[9] T. Luba, "Decomposition of multiple-valued functions," International Symposium on Multiple-Valued Logic, pp. 256-261, Bloomington, Indiana, May 1995.

[10] Y. Matsunaga, "An exact and efficient algorithm for disjunctive decomposition," SASIMI'98, pp. 44-50, Oct. 1998.

[11] R. Murgai, R. K. Brayton, and A. SangiovanniVincentelli, Logic Synthesis for Field-Programmable Gate Arrays, Kluwer, 1995.

[12] J. P. Roth and R. M. Karp, "Minimization over Boolean graphs," IBM Journal of Research and Development, pp. 227-238, April 1962.

[13] T. Sasao, "Application of multiple-valued logic to a serial decomposition of PLA's," International Symposium on Multiple-Valued Logic, pp. 264-271, Zangzou, China, May 1989.

[14] T. Sasao, "FPGA design by generalized functional decomposition," in (Sasao ed.) Logic Synthesis and Optimization, Kluwer Academic Publishers, 1993.

[15] T. Sasao and J. T. Butler, "On bi-decompositions of logic functions," ACM/IEEE International Workshop on Logic Synthesis, Tahoe City, California, May 1997.

[16] T. Sasao and M. Matsuura, "DECOMPOS: An integrated system for functional decomposition," $1998 \mathrm{In}$ ternational Workshop on Logic Synthesis, Lake Tahoe, June 1998.

[17] T. Sasao, "On a method to accelerate functional decompositions," Technical Reports of IEICE, VLD9858, pp. 103-109, Sept. 1998.

[18] C. Scholl and P. Molitor, "Communication based FPGA synthesis for multi-output Boolean functions," Asia and South Pacific Design Automation Conference, pp. 279-287, Aug. 1995.

[19] C. E. Shannon, "The synthesis of two-terminal switching circuits," Bell Syst. Tech. J. 28, 1, pp. 59-98, 1949.

[20] V. Y-S, Shen, A. C. Mckellar, and P. Weiner, "A fast algorithm for the disjunctive decomposition of switching functions," IEEE Trans. Comput., Vol. C20, No. 3, pp. 304-309, March 1971.

[21] W. Wan and M. A. Perkowski, "A new approach to the decomposition of incompletely specified multi-output functions based on graph coloring and local transformations and its application to FPGA mapping," European Design Automation Conference (Euro DAC), pp. 230-235, Sept. 1992.

[22] B. Wurth, K. Eckl, and K. Antreich, "Functional multiple-output decomposition: Theory and implicit algorithm," Design Automation Conf., pp. 54-59, June 1995 . 